\title{
Transient analysis of leaky Lamb waves with a semi-analytical finite element method
}

\section{AUTHOR(S):}

Inoue, Daisuke; Hayashi, Takahiro

\section{CITATION:}

Inoue, Daisuke ...[et al]. Transient analysis of leaky Lamb waves with a semi-analytical finite element method. Ultrasonics 2015, 62: 80-88

ISSUE DATE:

2015-09

URL:

http://hdl.handle.net/2433/216540

\section{RIGHT:}

(c) 2015. This manuscript version is made available under the CC-BY-NC-ND 4.0 license

http://creativecommons.org/licenses/by-nc-nd/4.0/; The full-text file will be made open to the public on 01 Septembe 2017 in accordance with publisher's 'Terms and Conditions for Self-Archiving'.; この論文は出版社版でありません。引 用の際には出版社版をご確認ご利用ください。; This is not the published version. Please cite only the published version. 


\title{
Transient analysis of leaky Lamb waves with a semi-analytical finite element method
}

Daisuke Inoue and Takahiro Hayashi

Graduate School of Engineering, Kyoto University, Kyoto, 615-8540, Japan

\begin{abstract}
We previously formulated a semi-analytical finite element technique for Lamb waves in a plate surrounded by fluids and investigated the dispersion curves and wave structures for leaky Lamb waves. Herein, this technique is extended to the calculation of transient responses both in a plate and in fluids for dynamic loading on the plate surface. To gain fundamental insights into guided wave inspection for a water-filled pipe or tank, guided waves generated upon transient loading of a flat plate water-loaded on one side were analyzed. The results show that a quasi-Scholte mode propagating at the plate-water interface is useful for the long-range inspection of a water-loaded plate because of its non-attenuation and minimal dispersion; moreover, this mode has superior generation efficiency in the low-frequency range, while it is localized near the plate-water interface at higher frequencies.
\end{abstract}

Keywords: Leaky Lamb waves, Semi-analytical finite element method, Non-destructive evaluation, transient responses

Contact author: Takahiro Hayashi: Graduate School of Engineering, Kyoto University C3 Kyotodaigaku-katsura, Nishikyo-ku, Kyoto, Japan +81-75-383-3797 (phone\&fax) ｅ-mail: hayashi@kuaero.kyoto-u.ac.jp 


\section{Introduction}

Elastodynamic problems such as ultrasonic propagation in solid media have frequently been solved numerically using general-purpose modeling techniques such as the finite difference method (FDM) [1, 2], finite element method (FEM) [3], and boundary element method (BEM) [4], where the continuum media are discretized into small subdivisions. Each modeling technique has advantages and disadvantages, and therefore, many extended techniques have already been developed to overcome their shortcomings. For example, the finite difference time domain (FDTD) method $[5,6]$ and elastodynamic finite integration technique (EFIT) [7] can be considered extended FDMs. FDTD was originally used for solving electric and magnetic fields in Maxwell's equation alternately. In elastodynamic problems, the velocity and stress fields are solved by the leap-flog algorithm. EFIT can be applied to inhomogeneous and/or curved regions by introducing the concept of a finite integration technique. An extended FEM (XFEM) [8] and spectral FEM [9] were developed as extended FEM techniques. Semi-analytical techniques with a Green's function such as BEM include CSM [10], MMP [11], and DPSM [12]. These extended BEMs can be considered extended analytical solutions because Green functions are a type of analytical solution.

We also have studied an extended technique of the FEM, called a semi-analytical finite element (SAFE) method [13-17]. In the SAFE, only a cross-section of an elongated structure is divided into small elements and displacement distributions in the longitudinal direction are expressed in terms of orthogonal functions. Consequently, calculation time and memory are significantly reduced especially in guided wave calculation. In addition, since the displacement field is expressed as a summation of guided wave modes, modal analysis can be carried out in a straightforward manner [13-25].

Cheung [26, 27] developed a special FEM for the static strain analysis of plate-like 
structures such as a box girder and folded plate in their early works; in this method, displacement distributions in the longitudinal direction were expressed as an orthogonal function. Subsequently, many studies have reported analogous techniques for elongated structures; these are called the semi-analytical finite element (SAFE) method by Zienkiewicz [28]. Similar semi-analytical techniques have been applied to dynamic problems such as vibration and wave propagation in the early stage. For example, Dong and Nelson calculated the frequency spectra of wavenumbers of guided wave modes in laminated plates using an extended Ritz method [29]. Datta et al. [30] and Kausel [31] obtained the frequency spectra of wavenumbers in a complex domain and discussed non-propagating as well as propagating modes. Gavrić [32], Gry [33], Thompson et al. [34] developed SAFE techniques for a bar with an arbitrary cross section for the vibration analyses of railway rails. These studies pertained to eigenmode analyses and dispersion curves. Liu and Achenbach [35] presented the time domain response for transient loading on a laminated plate. Hayashi [13-16] derived the group velocity dispersion curves required in guided wave inspection and visualized the guided wave motion by obtaining transient waves in the structure. Recently, Loveday et al. [18] and Mazzotti et al. [19] extended the SAFE technique to a bar with axial loading and a viscoelastic waveguide.

SAFE techniques have been widely used with the progress of guided wave inspection. However, most SAFE calculations are performed under the assumption of traction-free boundaries without the consideration of energy leakage to the surrounding media. Castaings and Lowe [17], Fan et al. [18], Treyssède et al. [19], and Nguyen and Treyssède [20] succeeded in modeling leaky guided waves by introducing absorbing layers and perfectly matched layers around external fluids. Mazzoti et al. developed a combination SAFE and BEM technique [24, 25]. These studies provided important results such as the dispersion curves and wave structures of guided waves in a bar with an external fluid, 
embedded solid plates, and embedded helical structures. The authors [17], too, have derived dispersion curves and attenuation curves for a plate loaded with a fluid using the characteristic that a harmonic plane leaky wave propagates at the velocity of the fluid for a single Lamb wave mode, and they discussed the vibration distributions for several modes.

Based on our previous study [17], this study derives the displacement responses in a plate cross section and external fluid regions for external harmonic point loading and calculates the time-domain waveforms by the inverse Fourier transform of the frequency-domain data. The time domain waveforms help our understanding of the generation mechanism of guided wave modes by external loading and the variations of waveforms due to the superposition of guided wave modes that cannot be understood in frequency domain analyses. To gain fundamental insights into the nondestructive evaluation of water-filled tanks and pipes, we investigate the wave propagation in a plate loaded with water on a single side.

\section{Semi-analytical finite element method for leaky Lamb waves}

\subsection{Frequency response for a point harmonic loading on a surface of a plate}

Since the extension of Ref. [17] yields transient responses for external dynamic loading, this section presents the formulation avoiding overlap with equations in Ref. [17]. Consider that time-harmonic point loadings $F_{1} \delta(z)$ and $F_{2} \delta(z)$ are applied at $z=0$ on surfaces of a plate in contact with semi-infinite fluid media of the common wave velocity ${ }^{c_{L 1}}$ and different density $\rho_{1}$ and $\rho_{2}$, where $y$ and $z$ are the thickness and longitudinal directions, respectively (Fig. 1). Letting a wavenumber of Lamb wave in the $+z$ direction be $\xi_{z}$ and a nodal displacement vector in the wavenumber-frequency domain with $2 \mathrm{~N}$ elements on $N$ line nodes be $\mathbf{U}$, the governing equation becomes

$$
\left(\mathbf{K}_{1}+i \xi_{z} \mathbf{K}_{2}+\xi_{z}^{2} \mathbf{K}_{3}-\omega^{2} \mathbf{M}\right) \mathbf{U}=\mathbf{F}^{\text {fluid }}+\mathbf{F}^{\text {ext }} \text {, }
$$


where $\mathbf{K}_{1}, \mathbf{K}_{2}, \mathbf{K}_{3}$ and $\mathbf{M}$ are known $2 N \times 2 N$ matrices calculated from material properties and thickness of the layered elements, $\mathbf{F}^{\text {fluid }}$ is a nodal force vector that acts on the plate surfaces from the fluid, $\mathbf{F}^{\text {ext }}$ is a nodal force vector by external dynamic loadings, and $\omega$ is an angular frequency. Using a $2 N \times 2 N$ matrix $\mathbf{Q}$ consisting of the fluid density $\rho_{1}, \rho_{2}$ and a wavenumber in the $y$ direction of harmonic wave radiating in fluids $\xi_{y}$ and a nodal displacement vector $\mathbf{U}, \mathbf{F}^{\text {fluid }}$ can be written as

$$
\mathbf{F}^{\text {fluid }}=\frac{\omega^{2}}{\xi_{y}} \mathbf{Q U}
$$

If external forces act on the plate surfaces in the thickness direction, the external nodal force vector becomes

$$
\mathbf{F}^{e x t}=\left(\begin{array}{llllll}
-F_{1} & 0 & \cdots & 0 & F_{2} & 0
\end{array}\right)^{T} .
$$

The governing equation (1) is rewritten as

$$
\left(\mathbf{K}_{1}+i \xi_{z} \mathbf{K}_{2}+\xi_{z}{ }^{2} \mathbf{K}_{3}-\omega^{2} \mathbf{M}-\frac{\omega^{2}}{\xi_{y}} \mathbf{Q}\right) \mathbf{U}=\mathbf{F}^{e x t}
$$

In the Ref. [17], the nonlinear eigenvalue problem of eq. (4) was linearized for $\mathbf{F}^{e x t}=0$ using the characteristics of leaky Lamb waves into the following form as,

$$
\left(\mathbf{A}-\xi_{y} \mathbf{B}\right) \mathbf{X}=0
$$

where $\mathbf{A}$ and $\mathbf{B}$ are $(4 N+2) \times(4 N+2)$ matrices consisting of known matrices $\mathbf{K}_{1}, \mathbf{K}_{2}, \mathbf{K}_{3}$, $\mathbf{M}, \mathbf{Q}$, and $\mathbf{X}$ is an unknown $4 N+2$ vector. Denoting the $y$ and $z$ components of the nodal displacement vector $\mathbf{U}$ by $\mathbf{U}_{y}$ ( $N$ vector) and $\mathbf{U}_{z}$ ( $N$ vector), respectively, and the $y$ displacement components on the lower and upper surfaces by $U_{y}{ }^{1}$ and $U_{y}{ }^{N}$, the unknown vector $\mathbf{X}$ is written as 


$$
\mathbf{X}=\left(\begin{array}{c}
\xi_{y}{ }^{2} \mathbf{U}_{y} \\
\xi_{y}{ }^{2} \xi_{z} \mathbf{U}_{z} \\
\xi_{y} \mathbf{U}_{y} \\
\xi_{y} \xi_{z} \mathbf{U}_{z} \\
U_{y}{ }^{1} \\
U_{y}{ }^{N}
\end{array}\right) .
$$

Solving the linear eigenvalue problem with respect to $\xi_{y}$ provides $4 N+2$ eigenvalues. The eigenvalues correspond to $2 N$ outgoing modes, $2 N$ incoming modes and two quasi-Scholte modes. Moreover, using the following relationship between the wavenumbers

$$
\xi_{y}^{2}+\xi_{z}^{2}=\xi_{f}^{2}, \xi_{y m}^{2}+\xi_{z m}^{2}=\xi_{f}^{2}
$$

$2 N$ pairs of $\pm \xi_{z}$ are obtained, where $\xi_{f}\left(=\omega / c_{L 1}\right)$ is the wavenumber of a plane harmonic wave in the fluid. The pair of wavenumber $\pm \xi_{z}$ stands for forward and backward waves. The solutions are classified as shown in Fig.2.

Following the form of Eq. (6), the right eigenvector for the $m$ th mode is written as

$$
\mathbf{V}^{R}{ }_{m}=\left(\begin{array}{c}
\xi_{y m}{ }^{2} \boldsymbol{\varphi}_{m y} \\
\xi_{y m}{ }^{2} \xi_{z m} \boldsymbol{\varphi}_{m z} \\
\xi_{y m} \boldsymbol{\varphi}_{m y} \\
\xi_{y m} \xi_{z m} \boldsymbol{\varphi}_{m z} \\
\varphi_{m y}{ }^{1} \\
\varphi_{m y}{ }
\end{array}\right)
$$

Now, consider the solution of Eq. (4) for harmonic loading at $z=0$. Like our previous study ${ }^{[17]}$, Eq. (4) is rewritten in the form of an eigen equation with respect to $\xi_{y}$ as

$$
\left(\mathbf{A}-\xi_{y} \mathbf{B}\right) \mathbf{X}=\tilde{\mathbf{F}}^{e x t}, \quad \tilde{\mathbf{F}}^{e x t}=\left(\begin{array}{c}
\mathbf{F}^{e x t} \\
0
\end{array}\right)
$$

The solution $\mathbf{X}$ of Eq. (9) can be expressed as a linear sum of right eigenvectors $\mathbf{v}_{m}^{R}$ that are obtained from Eq. (5) 


$$
\mathbf{X}=\sum_{m=1}^{4 N+2} \alpha_{m} \mathbf{v}_{m}^{R}
$$

Substituting Eq. (10) into Eq. (9) and multiplying a left eigenvector $\mathbf{v}^{L}{ }_{n}$ of Eq. (5) from the left provide

$$
\mathbf{v}_{n}^{L}\left(\mathbf{A}-\xi_{y} \mathbf{B}\right) \mathbf{X}=\sum_{m=1}^{4 N+2}\left[\alpha_{m}\left(\mathbf{v}_{n}^{L} \mathbf{A} \mathbf{v}_{m}^{R}-\xi_{y} \mathbf{v}_{n}^{L} \mathbf{B} \mathbf{v}_{m}^{R}\right)\right]
$$

Now, using the relationships as

$$
\begin{array}{ll}
\mathbf{v}_{n}^{L} \mathbf{A} \mathbf{v}{ }^{R}{ }_{m}=\mathbf{v}_{n}^{L} \mathbf{B} \mathbf{v}^{R}{ }_{m}=0 & \text { if } m \neq n \\
\mathbf{v}^{L}{ }_{n} \mathbf{A} \mathbf{v}^{R}{ }_{m}=\xi_{y m} \mathbf{v}_{n}^{L} \mathbf{B} \mathbf{v}^{R}{ }_{m} & \text { if } m=n,
\end{array}
$$

Eq. (11) becomes

$$
\begin{aligned}
\mathbf{v}^{L}{ }_{n}\left(\mathbf{A}-\xi_{y} \mathbf{B}\right) \mathbf{X} & =\alpha_{n}\left(\mathbf{v}^{L}{ }_{n} \mathbf{A} \mathbf{v}^{R}{ }_{n}-\xi_{y} \mathbf{v}^{L}{ }_{n} \mathbf{B} \mathbf{v}^{R}{ }_{n}\right) \\
& =\alpha_{n}\left(\xi_{y n}-\xi_{y}\right) \mathbf{v}^{L}{ }_{n} \mathbf{B} \mathbf{v}^{R} .
\end{aligned}
$$

Because the right-hand side of Eq. (9) becomes $\mathbf{v}_{n}^{L} \tilde{\mathbf{F}}^{\text {ext }}$, the following equation holds

$$
\alpha_{n}\left(\xi_{y n}-\xi_{y}\right) \mathbf{v}_{n}^{L} \mathbf{B} \mathbf{v}^{R}{ }_{n}=\mathbf{v}_{n}^{L} \tilde{\mathbf{F}}^{e x t}
$$

and the amplitude of the $n$th mode $\alpha_{n}$ can be obtained as,

$$
\alpha_{n}=\frac{-\mathbf{v}_{n}^{L} \tilde{\mathbf{F}}^{e x t}}{\left(\xi_{y}-\xi_{y n}\right) \mathbf{v}^{L}{ }_{n} \mathbf{B v}_{n}{ }_{n}} .
$$

Considering Eqs. (6) and (8), extracting the first to the $N$ th elements as well as the $(N+1)$ th to the 2Nth elements in Eq. (10) gives

$$
\xi_{y}{ }^{2} \mathbf{U}_{y}=\sum_{m=1}^{4 N+2} \alpha_{m} \xi_{y m}{ }^{2} \boldsymbol{\varphi}_{m y}, \quad \xi_{y}{ }^{2} \xi_{z} \mathbf{U}_{z}=\sum_{m=1}^{4 N+2} \alpha_{m} \xi_{y m}{ }^{2} \xi_{z m} \boldsymbol{\varphi}_{m z}
$$

and introducing the following variables

$$
\alpha_{m}^{\prime}=\frac{-\mathbf{v}^{L}{ }_{m} \tilde{\mathbf{F}}^{e x t}}{\mathbf{v}_{m}^{L} \mathbf{B v}^{R}{ }_{m}},
$$


Eq. (16) is rewritten as

$$
\mathbf{U}_{y}=\sum_{m=1}^{4 N+2} \alpha_{m}^{\prime} \frac{\xi_{y m}{ }^{2}}{\left(\xi_{y}-\xi_{y m}\right) \xi_{y}{ }^{2}} \boldsymbol{\varphi}_{m y}, \quad \mathbf{U}_{z}=\sum_{m=1}^{4 N+2} \alpha_{m}{ }^{\prime} \frac{\xi_{y m}{ }^{2} \xi_{z m}}{\left(\xi_{y}-\xi_{y m}\right) \xi_{y}{ }^{2} \xi_{z}} \boldsymbol{\varphi}_{m z} .
$$

Using Eq. (7), Eq. (18) becomes

$$
\begin{aligned}
& \mathbf{U}_{y}=\sum_{m=1}^{4 N+2} \alpha_{m}^{\prime} \frac{\left(\xi_{y}+\xi_{y m}\right) \xi_{y m}{ }^{2}}{\left(\xi_{z}+\xi_{z m}\right)\left(\xi_{z}-\xi_{z m}\right)\left(\xi_{z}+\xi_{f}\right)\left(\xi_{z}-\xi_{f}\right)} \boldsymbol{\varphi}_{m y} \\
& \mathbf{U}_{z}=\sum_{m=1}^{4 N+2} \alpha_{m}^{\prime} \frac{\left(\xi_{y}+\xi_{y m}\right) \xi_{y m}{ }^{2} \xi_{z m}}{\left(\xi_{z}+\xi_{z m}\right)\left(\xi_{z}-\xi_{z m}\right)\left(\xi_{z}+\xi_{f}\right)\left(\xi_{z}-\xi_{f}\right) \xi_{z}} \boldsymbol{\varphi}_{m z}
\end{aligned}
$$

Because the nodal displacement vectors $\mathbf{U}_{y}$ and $\mathbf{U}_{z}$ in Eq. (19) are the wavenumber-domain representation, the space-domain nodal displacement vectors are the inverse Fourier transform of $\mathbf{U}_{y}$ and $\mathbf{U}_{z}$ as

$$
\mathbf{u}_{y}=\int_{-\infty}^{\infty} \mathbf{U}_{y} \exp \left(i \xi_{z} z\right) d \xi_{z}, \quad \mathbf{u}_{z}=\int_{-\infty}^{\infty} \mathbf{U}_{z} \exp \left(i \xi_{z} z\right) d \xi_{z}
$$

The infinite integration can be calculated by the residue theorem with respect to the contour as shown in Fig. 3. Four or five poles $\pm \xi_{z m}, \pm \xi_{f}$ and 0 exist for each $m$ in Eq. (19a) and (19b). If the poles are located on the real axis, the integration paths are selected to obtain physically reasonable solutions. Namely, $\pm \xi_{f}$ and 0 should not be within the interior of the integration path. If $\pm \xi_{\text {zm }}$ are real, a positive propagating mode (namely $c_{g m}>0$ ) should be included in $Z>0$ as shown in solid closed curves, while a negative propagating mode (namely $C_{g m}<0$ ) should be included in $Z<0$ as shown in dashed closed curves, where the group velocity of the $m$ th mode $c_{g m}$ is defined as $\partial \omega / \partial \xi_{z m}$ for real $\xi_{z m}$. Because $\pm \xi_{z m}$ can be selected positive and negative values arbitrarily, assuming $+\xi_{z m}$ is the pole that should be included in the closed path for $z>0$ gives the 
nodal displacement vector in the space domain as

$$
\begin{aligned}
\mathbf{u}_{y} & =2 \pi i \lim _{\substack{\left.\xi_{z} \rightarrow \xi_{z m} \\
\xi_{y} \rightarrow \xi_{y m}\right)}} \sum_{m=1}^{4 N+2} \alpha_{m}^{\prime} \frac{\left(\xi_{y}+\xi_{y m}\right) \xi_{y m}{ }^{2}}{\left(\xi_{z}+\xi_{z m}\right)\left(\xi_{z}+\xi_{f}\right)\left(\xi_{z}-\xi_{f}\right)} \boldsymbol{\varphi}_{m y} \exp \left(i \xi_{z m} z\right) \\
& =2 \pi i \sum_{m=1}^{4 N+2} \alpha_{m}^{\prime} \frac{-\xi_{y m}}{\xi_{z m}} \boldsymbol{\varphi}_{m y} \exp \left(i \xi_{z m} z\right) \\
\mathbf{u}_{z} & =2 \pi i \lim _{\substack{\xi_{z} \rightarrow \xi_{z m} \\
\left(\xi_{y} \rightarrow \xi_{y m}\right)}} \sum_{m=1}^{4 N+2} \alpha_{m}^{\prime} \frac{\left(\xi_{y}+\xi_{y m}\right) \xi_{y m}{ }^{2} \xi_{z m}}{\left(\xi_{z}+\xi_{z m}\right)\left(\xi_{z}+\xi_{f}\right)\left(\xi_{z}-\xi_{f}\right) \xi_{z}} \boldsymbol{\varphi}_{m z} \exp \left(i \xi_{z m} z\right) \\
& =2 \pi i \sum_{m=1}^{4 N+2} \alpha_{m} \frac{-\xi_{y m}}{\xi_{z m}} \boldsymbol{\varphi}_{m z} \exp \left(i \xi_{z m} z\right) .
\end{aligned}
$$

Summarizing Eqs. (21a) and (21b) gives

$$
\mathbf{u}=\left(\begin{array}{l}
\mathbf{u}_{y} \\
\mathbf{u}_{z}
\end{array}\right)=\sum_{m=1}^{4 N+2} C^{F}{ }_{m} \boldsymbol{\varphi}_{m} \exp \left(i \xi_{z m} z\right), C^{F}{ }_{m}=2 \pi i \frac{\mathbf{v}^{L}{ }_{m} \tilde{\mathbf{F}}^{e x t}}{\mathbf{v}_{m}^{L} \mathbf{B v}^{R}{ }_{m}} \frac{\xi_{y m}}{\xi_{z m}} .
$$

In $z<0$, the displacement field can be obtained by replacing $\xi_{z m}$ by $-\xi_{z m}$ as

$$
\mathbf{u}=\sum_{m=1}^{4 N+2} C^{B}{ }_{m} \boldsymbol{\varphi}_{m} \exp \left(-i \xi_{z m} z\right), C^{B}{ }_{m}=-2 \pi i \frac{\mathbf{v}^{L}{ }_{m} \tilde{\mathbf{F}}^{e x t}}{\mathbf{v}^{L}{ }_{m} \mathbf{B} \mathbf{v}^{R}{ }_{m}} \frac{\xi_{y m}}{\xi_{z m}} .
$$

\subsection{Displacement field in fluids}

The displacement field in external fluids can be derived from out-of-plane displacement on a plate surface. For example, considering a harmonic wave of the $m$ th mode and letting the displacement in the $y$ direction on the upper surface $\left(y=y_{2}\right)$ at $z=0$ be $U_{y m}^{y=y_{2}}$, the displacement in the $z$ direction in the fluid can be represented as $\frac{\xi_{z m}}{\xi_{y m}} U_{y m}^{y=y_{2}}$ and then a displacement vector at an arbitrary position in the upper fluid region $y>y_{2}$ for the $m$ th mode becomes 


$$
\mathbf{u}_{m}=\left(\begin{array}{c}
1 \\
\xi_{z m} / \xi_{y m}
\end{array}\right) U_{y m}^{y=y_{2}} \exp \left\{i \xi_{z m} z+i \xi_{y m}\left(y-y_{2}\right)\right\},
$$

where $U_{y m}^{y=y_{2}}$ is obtained from Eq. (22). The displacement vector at an arbitrary position in the fluid region can be represented as the superposition of Eq. (24).

$$
\mathbf{u}=\sum_{m=1}^{2 N+2} \mathbf{u}_{m}
$$

\subsection{Verification of accuracy of the SAFE calculations}

The formulations described above provide the displacement responses at an arbitrary position for a harmonic point loading. To verify the accuracy of the SAFE calculation, the energy balance of waves propagating in and leaking out from a plate is calculated.

An aluminum alloy plate of thickness $d$ having the longitudinal velocity $6310 \mathrm{~m} / \mathrm{s}$, transverse velocity $3140 \mathrm{~m} / \mathrm{s}$ and density $2700 \mathrm{~kg} / \mathrm{m}^{3}$ and water having the sound velocity $1485 \mathrm{~m} / \mathrm{s}$ and density $1000 \mathrm{~kg} / \mathrm{m}^{3}$ are used as a waveguide and a leaky medium in this study. As shown in Fig. 4 (a), when a point source is located at $z=0$, the vibration energy flows from the cross-sectional boundary $\Gamma_{1}$ at $z=0$ and leaks out from the other boundaries $\Gamma_{2}, \Gamma_{3}$ and $\Gamma_{4}$. Letting displacements in the $y$ and $z$ directions at an arbitrary position on the boundary $\Gamma_{1}$ be $u_{y}, u_{z}$ and the normal stress and the shear stress on the boundary be $\sigma_{z}, \tau_{y z}$, the time-averaged energy flux across $\Gamma_{1}$ is represented as

$$
E_{1}=\operatorname{Re}\left[\frac{-i \omega}{2} \int_{\Gamma_{1}}\left(u_{z} \cdot \sigma_{z} *+u_{y} \cdot \tau_{y z} *\right) d \Gamma_{1}\right],
$$

where * denotes complex conjugate and the integration on the cross-sectional boundary $\Gamma_{1}$ was calculated by numerical integration. Similarly, the time-averaged energy flows $E_{2}, E_{3}$, $E_{4}$ are calculated. Figure 5 (a) shows that energy ratio of outflow to inflow $\left(E_{2}+E_{3}+E_{4}\right) / E_{1}$ is within $1.00 \pm 0.01$ in the frequency range for various numbers of SAFE elements $M=8$, 
16 and 32, which indicates that the SAFE calculations were executed with very high accuracy from the view point of energy balance.

In the SAFE calculation, each leaky medium can be replaced with a vacuum region by setting the density of the region to zero ${ }^{[17]}$. When the upper region is a vacuum $\left(\rho_{2}=0\right)$ as shown in Fig. 4 (b), the energy ratio $\left(E_{2}+E_{3}\right) / E_{1}$ is also about 1.00 as shown in Fig. 5 (b). These results also show that the SAFE calculation is done with high accuracy.

\section{Analyses of transient responses for non-destructive inspection of a water-loaded} plate

The displacement field calculated in the frequency domain can be converted into one in time domain by the inverse Fourier transform. Thus, transient waves at arbitrary positions can be calculated and wave propagation can be simulated both in a plate and fluids.

Considering the guided wave inspection of a tank and a pipe filled with water as a typical example, transient waves in a plate loaded with water as shown in Fig. 6 are analyzed in this section. Since guided waves in a pipe and a plate have many similar features, simulations of leaky Lamb wave propagation in a water-loaded plate are useful to obtain basic insight into the physical phenomenon as a first step. Material constants of a plate and water are the same as in the previous section and the number of layered elements is $M=32$.

A transient load is acting at $z=0$ normal to the surface of a vacuum side as

$$
s(\bar{t})=\exp \left(-2 \pi i \bar{f}_{c} \bar{t}-\frac{\bar{t}^{2}}{2 \bar{\sigma}^{2}}\right),
$$

where - denotes non-dimensional parameters. The non-dimensional time $\bar{t}$, non-dimensional center frequency $\bar{f}_{c}$ and non-dimensional standard deviation of 
Gaussian distribution $\bar{\sigma}$ are defined using plate thickness $d$ and transverse velocity $c_{T}$ as

$$
\bar{t}=t c_{T} / d, \bar{f}_{c}=f_{c} d / c_{T}, \bar{\sigma}=\sigma c_{T} / d
$$

The other parameters appearing below are also normalized by $d, d / c_{T}$, and $c_{T} / d$ for parameters having the dimension of distance, for those of the dimension of time and for those of the dimension of frequency, respectively.

Figure 7 shows the waveforms of input loadings used in the transient wave analyses. We investigate four different central frequencies of $\bar{f}_{c}=0.125,0.25,0.5,1$ with $\bar{\sigma}=\frac{\sqrt{2}}{0.3 \bar{f}_{c}}$. Frequency spectra of the input loadings are illustrated in Fig. 8 (a).

Figures 8 (a) - (c) are dispersion curves for phase velocity, group velocity and attenuation calculated with the SAFE for the aluminum plate water-loaded on the single surface. (a) and (b) show only propagating modes having the imaginary part of wavenumber smaller than $\operatorname{Im}\left(\bar{\xi}_{z m}\right)=1$. Here we define the group velocity $c_{g}$ as $\partial \omega / \partial \operatorname{Re}\left(\xi_{z}\right)$, because the group velocity calculated from time-averaged Poyting vector and energy velocity flux are almost identical to $\partial \omega / \partial \operatorname{Re}\left(\xi_{z}\right)$ within small attenuation $\operatorname{Im}\left(\xi_{z m}\right)$ range [36]. We can estimate the degree of velocity dispersion from the dispersion curves and the frequency spectra of input loadings.

Figures 9 - 12 are the time-variation of displacement in the $y$ direction at six positions $\bar{z}(=z / d)=0,100,200,300,400,500$ on the lower plate surface contacting to water, and the distributions of displacement in the $y$ direction at a representative time. Because the amplitude is much larger at $\bar{z}=0$ than the other distances in (a), the waveforms were magnified by the numerical factor indicated. Additionally, in (a), the expected arrival times of different modes are indicated by dashed lines that were calculated from the group velocities at $\bar{f}=\bar{f}_{c}$. Note that Lamb wave modes are now called S or A in 
accordance with Lamb waves in a plate with traction-free boundaries. Strictly speaking, each mode does not have symmetric or anti-symmetric displacement distributions with respect to the center-line of a plate because the boundary conditions on the upper and lower surfaces are not identical. However, as displacement distributions maintain approximately symmetric or anti-symmetric forms and the dispersion curves of phase and group velocities are only slightly different from those in a plate with traction-free boundaries, we call them symmetric (S) or anti-symmetric (A) modes in this paper. Apart from S and A modes, the mode with the velocity close to water appearing in the phase and group velocity dispersion curves is an interface wave propagating at the interface between a plate and fluid, called a quasi-Scholte mode.

In the lowest frequency band of $\bar{f}_{c}=0.125$, three propagating modes exist; $\mathrm{S}_{0}, \mathrm{~A}_{0}$ and a quasi-Scholte mode. In Fig. 9, the quasi-Scholte mode appears in large amplitude and very small $S_{0}$ mode can be seen at $\bar{z} \geq 100$, while the $A_{0}$ mode disappears. This can be explained by the characteristics that both $\mathrm{S}_{0}$ and quasi-Scholte modes have small attenuation and dispersion in the frequency band around $\bar{f}_{c}=0.125$. For example, the attenuation coefficient $\operatorname{Im}\left(\bar{\xi}_{z m}\right)$ of the $\mathrm{S}_{0}$ mode at $\bar{f}_{c}=0.125$ is $6.77 \times 10^{-4}$, and so the amplitude of harmonic $S_{0}$ mode at $\bar{z}=500$ is $e^{-6.77 \times 10^{-4} \times 500}=0.713$ times of its amplitude at $\bar{z}=0$. In addition, the group velocity dispersion curve of the $\mathrm{S}_{0}$ mode in Fig. 8 (b) represents that it is a weakly dispersive mode in this frequency band. The quasi-Scholte mode can also propagate over long distances because the attenuation coefficient is zero over the whole frequency range and its dispersion is small beyond the frequency region of $\bar{f}_{c}=0.125$. It should be noted that the quasi-Scholte wave in the low frequency range has a dispersive characteristic because the interface wave is affected by the opposite surface of the plate. A Scholte mode at the interface between semi-infinite solid and fluid media is generally known 
to be non-attenuated and non-dispersive, which corresponds to the high frequency range in this study. On the other hand, we cannot find a waveform of the $A_{0}$ mode in Fig. 9 (a). Out-of-plane vibration, an $\mathrm{A}_{0}$ mode, is generated in a plate significantly when an out-of-plane loading acts on a surface of a plate with no fluids. However, for a water-loaded plate, the $\mathrm{A}_{0}$ mode disappeared due to its large attenuation. In the attenuation curves of Fig. 8 (c), the attenuation coefficient $\operatorname{Im}\left(\bar{\xi}_{z m}\right)$ of the $\mathrm{A}_{0}$ mode indicates 0.144 , which means that the $A_{0}$ mode may disappear over $\bar{Z} \geq 100$ because the $A_{0}$ mode amplitude at $\bar{Z}=100$ becomes $e^{-0.144 \times 100}=5.72 \times 10^{-7}$ times of that at $\bar{z}=0$. On the contrary, since all propagating and non-propagating modes are superposed at $\bar{z}=0$ just under the point source, a large waveform can be observed at the opposite surface of the source. It should be noted that the waveform at $\mathrm{z}=0$ in Fig. 9 (a) is reduced to $1 / 5$ of the others to recognize the waveforms in the same figure.

Figures 9 (b) and (c) are snapshots of displacement distribution in the range from $\bar{Z}=90$ to 110 for $\bar{f}_{c}=0.125$. The mesh deformation expresses the two-dimensional displacement field and the gray scale in the region means the vertical displacement $u_{y}$. Figure 9 (b) shows the snapshot at $\bar{t}=75$ when the $\mathrm{S}_{0}$ mode arrives at the region from $\bar{Z}=$ 90 to 110 . The vertical displacement in the plate region has the opposite phase at the upper and lower surfaces as shown in Fig. 9 (b) and the leaky waves of the $S_{0}$ mode form an oblique plane wave. These are typical features of leaky Lamb waves.

Figure 9 (c) is the snapshot at $\bar{t}=188$ when the quasi-Scholte mode arrives. Although a Scholte wave is known as an interface wave that concentrates at a solid-liquid interface in energy, the quasi-Scholte mode in the low frequency range accompanies vibration over the whole cross-section of the plate. Furthermore, the wavefront in water is vertical to the plate surface, because the phase velocity of the quasi-Scholte mode almost equals to the sound speed of water. 
Figure 10 (a) represents the $y$-directional displacement at the same six positions for $\bar{f}_{c}=0.25$. Similarly, the waveform at $\bar{z}=0$ is much larger than that of $\bar{z} \geq 100$, showing that the waveform contains the highly attenuated $\mathrm{A}_{0}$ mode and the other non-propagating modes together with the quasi-Scholte mode. And the $\mathrm{S}_{0}$ and quasi-Scholte modes can propagate over long distances like in the case of the low frequency range of $\bar{f}_{c}=0.125$. Unlike the waveforms at $\bar{f}_{c}=0.125$ shown in Fig. 9 (a), the wave packets in Fig. 10 (a) are more localized due to their higher frequency components and the nearly non-dispersive nature of quasi-Scholte mode. These features are useful in the non-destructive inspection. Figure 10 (b) shows that the $\mathrm{S}_{0}$ mode at $\bar{f}_{c}=0.25$ has vibration over the whole thickness like in Fig. 9 (b), while the quasi-Scholte mode in Fig. 10 (c) propagates with larger amplitude at the lower plate surface. This indicates that the quasi-Scholte mode is more effective for defect detection of the lower water-loaded surface.

Next, Fig. 11 (a) represents the $y$-directional displacement at the same six positions for $\bar{f}_{c}=0.5$. The $\mathrm{A}_{0}$ mode disappears over $\overline{\mathrm{Z}} \geq 100$ due to its large attenuation coefficient $\operatorname{Im}\left(\bar{\xi}_{\text {zm }}\right)=0.0795$ like in $\bar{f}_{c}=0.125$ and 0.25 . The $\mathrm{S}_{0}$ mode gradually becomes smaller as it propagates due to attenuation and dispersion. The quasi-Scholte mode cannot be clearly seen at $\bar{z}=100$ because of the superposition of $A_{1}$ mode that spreads widely. As they propagate, a sharp pulse of quasi-Scholte mode can be observed. This feature is caused by the facts that the $\mathrm{A}_{1}$ mode amplitude decreased by the dispersion and attenuation and that the quasi-Scholte mode retained its pulse shape due to non-dispersion and non-attenuation natures.

Figures 11 (b) and (c) are snapshots showing vibration distributions at the two points in Fig. 11 (a). Figure 11 (b) corresponds to $\bar{t}=424$ when the $A_{1}$ and quasi-Scholte modes overlapped at $\bar{Z}=190$ to 210 , and (c) to one at $\bar{t}=1058$ when the $\mathrm{A}_{1}$ mode 
disappeared and only the quasi-Scholte mode remains in the region of $\bar{z}=490$ to 510. In (b), we can see the oblique pattern of leaky wave of the $A_{1}$ mode as well as the vertical pattern of the quasi-Scholte mode. While in (c), we can observe only the quasi-Scholte mode that concentrates at the lower water-loaded surface of the plate.

Finally, we discuss the displacement at the six positions at $\bar{f}_{c}=1$ and the vibration distributions at $\bar{t}=179$ at $\bar{z}=190$ to 210 in Fig. 12. Only a single attenuated and dispersive mode can be seen in Fig. 12 (a). The displacement distribution in the plate shown in Fig. 12 (b) proves that the mode has a symmetric distribution with respect to the centerline of the plate. Judging from the attenuation curves, it is likely that the mode remaining even at $\bar{z}=$ 500 is the $S_{1}$ mode having small attenuation at around $\bar{f}_{c}=1.2$. The group velocity of the $S_{1}$ mode at $\bar{f}_{c}=1.2$ is slightly higher than one at the center frequency of $\bar{f}_{c}=1$. Therefore we can observe the wave packet slightly faster than the dashed line of $S_{1}$ mode.

From the calculation results shown above, when Lamb waves are generated in a plate water-loaded on a single surface by dynamic loads on the other vacuum surface, a quasi-Scholte mode is also excited. Because it is a non-attenuated and weakly dispersive wave which propagates over long distances, the quasi-Scholte mode is very effective for the non-destructive inspection of the plate loaded on one side by water. Although the quasi-Scholte mode can be excited on the water-loaded surface in the low frequency range such as $\bar{f}_{c}=0.125$ and 0.25 , it is not very sensitive to small defects at the water-loaded surface because its vibration energy spreads over the plate thickness. In contrast, in the high frequency range as $\bar{f}_{c}=0.5$ and 1 , the quasi-Scholte mode would be effective for such defects because the energy concentrates at the water-loaded surface. However, dynamic loading on the opposite surface results in poor generation efficiency. In summary, in order to use the quasi-Scholte wave in a long-range inspection, we need to find the appropriate 
frequency band by considering the generation efficiency and defect detectability besides its attenuation and dispersion.

\section{Conclusions}

We extended formulation of the semi-analytical finite element method for leaky Lamb waves so as to calculate transient responses of dynamic loading on the surfaces of a fluid-loaded plate. Using eigenvalues and eigenvectors obtained from an eigenvalue problem of a plate loaded with fluids, amplitudes of guided wave modes were calculated for dynamic point loading in frequency-wavenumber domains. Converting the displacements in frequency-wavenumber domains into those in time-space domains, we obtained transient responses at arbitrary positions. In order to gain basic insight into the guided wave inspection of water-filled pipes and tanks, transient responses in a flat plate that is loaded with water on a single surface and with dynamic force on the other vacuum surface were analyzed. The results showed that the quasi-Scholte mode at the plate-water interface propagates over long distances due to the features of non-attenuation and small dispersion. In the low frequency range, we can generate the quasi-Scholte mode effectively, but it accompanies vibration over the whole cross-section of a plate and is expected to be insensitive to small defects on a water-plate surface. On the other hand, the quasi-Scholte mode concentrates on the water-plate interface in the high frequency range and it is sensitive to defects on the inner surface, but we cannot generate it effectively by dynamic loading on the vacuum surface. It remains as a subject of future study to explore the optimal frequency range to achieve efficient generation and high defect-sensitivity of the Scholte mode.

\section{Acknowledgments}

The authors would like to thank Professor Shiro Biwa for his insightful comments and 
continuing support. This work was supported by JSPS KAKENHI Grant Number 26282094.

\section{References}

[1] K. Harumi, F. Suzuki, Y. Sato, Computer simulation of the nearfield for elastic wave in a solid half-space, J. Acoust. Soc. Am. 53 (1973) 660-664.

[2] L.J. Bond, A computer model of the interaction of acoustic surface waves with discontinuities, Ultrasonics. 17 (1979) 71-77.

[3] C. Zienkiewicz and R. L. Taylor, The finite element method, fifth edition, Butterworth-Heinemann, Oxford, 2000

[4] C. A. Brebbia, and J. Dominguez, Boundary elements: introductory course, WIT press, Southampton, 1992

[5] K. S. Kunz and R. J. Luebbers, Time domain method for electromagnetics, CRC press, Boca Raton, 1993

[6] Y. Hao, M. Sato, FDTD formulation for analysis of elastic wave fields in cylindrical coordinates, Japan Soc. Simul. Technol. 20 (2001) 60-68.

[7] F. Schubert, A. Peiffer, B. Köller, T. Sanderson, The elastodynamic finite integration technique for waves in cylindrical geometries, 104 (2000) 2604-2614.

[8] Z. Liu, J. Oswald, T. Belytschko, XFEM modeling of ultrasonic wave propagation in polymer matrix particulate/fibrous composites, Wave Motion. 50 (2013) 389-401.

[9] M. Palacz, M. Krawczuk, Analysis of longitudinal wave propagation in a cracked rod by the spectral element method, Comput. Struct. 80 (2002) 1809-1816.

[10] C. Rajamohon, and J. Raamachandran, Bending of anisotropic plates by chage simulation method, Advances in Engineering Software, 30 (1999) pp.363-373

[11] R. Ballisti, and C. Hafner, The multiple multipole method in electro- and magnetostatic problems, IEEE trans., magnetics, MAG-19 (1983) pp.2367-2370 
[12] D. Placko; and T. Kundu, A theoretical study of magnetic and ultrasonic sensors: dependence of magnetic potential and acoustic pressure on the sensor geometry. Advanced NDE for structural and biological health monitoring, Proceedings of SPIE. In: Kundu, T. (Ed.), SPIE’s 6th Annual International Symposium on NDE for Health Monitoring and Diagnostics, March 4-8, vol. 4335, Newport Beach, CA, pp. 52-62.

[13] T. Hayashi, K. Kawashima, Multiple reflections of Lamb waves at a delamination, Ultrasonics. 40 (2002) 193-197.

[14] T. Hayashi, W - J Song, J. L. Rose, Guided wave dispersion curves for a bar with an arbitrary cross-section, a rod and rail example. Ultrasonics. 41 (2003) 175-183.

[15] T. Hayashi, K. Kawashima, Z. Sun, J.L. Rose, Analysis of flexural mode focusing by a semianalytical finite element method., J. Acoust. Soc. Am. 113 (2003) 1241-1248.

[16] T. Hayashi, C. Tamayama, M. Murase, Wave structure analysis of guided wave in a bar with an arbitrary cross-section, Ultrasonics. 44 (1) (2006) 17-24.

[17] T. Hayashi, D. Inoue, Calculation of leaky Lamb waves with a semi-analytical finite element method., Ultrasonics. 54 (6) (2014) 1460 - 1469.

[18] P.W. Loveday, Semi-analytical finite element analysis of elastic waveguides subjected to axial loads., Ultrasonics. 49 (2009) 298-300.

[19] M. Mazzotti, a. Marzani, I. Bartoli, E. Viola, Guided waves dispersion analysis for prestressed viscoelastic waveguides by means of the SAFE method, Int. J. Solids Struct. 49 (2012) 2359-2372.

[20] M. Castaings, M. Lowe, Finite element model for waves guided along solid systems of arbitrary section coupled to infinite solid media., J. Acoust. Soc. Am. 123 (2008) 696-708.

[21] Z. Fan, M.J.S. Lowe, M. Castaings, C. Bacon, Torsional waves propagation along a waveguide of arbitrary cross section immersed in a perfect fluid., J. Acoust. Soc. Am. 
124 (2008) 2002-2010.

[22] F. Treyssède, K.L. Nguyen, a.-S. Bonnet-BenDhia, C. Hazard, Finite element computation of trapped and leaky elastic waves in open stratified waveguides, Wave Motion. 51 (2014) 1093-1107.

[23] K.L. Nguyen, F. Treyssède, Numerical investigation of leaky modes in helical structural waveguides embedded into a solid medium., Ultrasonics. 57 (2015) 125-34.

[24] M. Mazzotti, I. Bartoli, a. Marzani, E. Viola, A coupled SAFE-2.5D BEM approach for the dispersion analysis of damped leaky guided waves in embedded waveguides of arbitrary cross-section, Ultrasonics. 53(7) (2013) 1227 - 1241.

[25] M. Mazzotti, I. Bartoli, G. Castellazzi, a Marzani, Computation of leaky guided waves dispersion spectrum using vibroacoustic analyses and the Matrix Pencil Method: a validation study for immersed rectangular waveguides., Ultrasonics. 54 (2014) 1895-1898.

[26] Y. K. Cheung, Finite strip method analysis of elestic slabs, J. of Eng. Mech, ASCE 94, (1968) 1365-1378

[27] M. S. Cheung and Y. K. Cheung,Natural vibrations of thin, flat-walled structures with different boundary conditions, J. of Sound and Vibration 18, (1971) 325-337.

[28] C. Zienkiewicz and R. L. Taylor, The finite element method, fifth edition, Butterworth-Heinemann, Oxford, 2000

[29] R.B. Dong, S. B., Nelson, On natural vibrations and waves in laminated orthotropic plates, J. Appl. Mech. 39 (1972) 739-745.

[30] R.L. Datta, S. K., Shah A. H., Bratton, T. Chakraborty, Wave propagation in laminated composite plates, J. Acoust. Soc. Am. 83 (1988) 2020-2026.

[31] E. Kausel, Wave propagation in anisotropic layered media, Int. J. Numer. Methods Eng. 23 (1986) 1567-1578.

[32] L. Gavrić, Computation of propagative waves in free rail using a finite element 
technique, Journal of s Sound Vibration. 185 (1995) 531-543.

[33] L. Gry, Dynamic modelling of railway track based on wave propagation, J. Sound Vibration. 195 (3) (1996) 477-505.

[34] D. J. Thompson, C.J.C. Jones, Sound radiation from a vibrating railway wheel, J. Sound Vibration. 253 (2) (2002) 401-419.

[35] G.R. Liu and J.D. Achenbach, A Strip element method to analyze wave scattering by cracks in anisotropic laminated plates, J. Appl. Mech. 62, (1995) 607-613.

[36] A. Bernard, M.J.S. Lowe, M. Deschamps, Guided waves energy velocity in absorbing and non-absorbing plates, J. Acoust. Soc. Am. 110 (2001) 186-196. 


\section{Figure Captions}

Fig. 1. Layered elements and leaky media for the SAFE calculation.

Fig. 2. Classification of eigenvalues obtained from Eq. (5).

Fig. 3. Integral paths in Eq. (20).

(a) $\xi_{z m}$ is real and (b) $\xi_{z m}$ is complex.

Fig. 4. Regions for verifying calculation accuracy of the SAFE calculations.

(a) A plate loaded with water on both surfaces and (b) a plate loaded with water on a single surface.

Fig. 5. Ratio of outflow energy to inflow energy.

(a) A plate loaded with water on both surfaces and (b) a plate loaded with water on a single surface.

Fig. 6. Calculation region for transient wave analysis.

Fig. 7. Waveforms of dynamic loading on the upper surface at $z=0$ used in this study.

Fig. 8. Dispersion curves for an aluminum plate with water on a single surface.

(a) Phase velocity , (b) group velocity and (c) attenuation.

Fig. 9. Waveforms at six positions and displacement distributions for input loading of $\bar{f}_{c}=0.125$. (a) Displacement in the $y$ direction, (b) snapshot at $\bar{t}=75$ and $\bar{z}=90-110$, 
and (c) snapshot at $\bar{t}=188$ and $\bar{Z}=90-110$.

Fig. 10. Waveforms at six positions and displacement distributions for input loading of $\bar{f}_{c}=0.25$. (a) Displacement in the $y$ direction, (b) snapshot at $\bar{t}=119$ and $\bar{Z}=190-210$, and (c) snapshot at $\bar{t}=424$ and $\bar{Z}=190-210$.

Fig. 11. Waveforms at six positions and displacement distributions for input loading of $\bar{f}_{c}=0.5$. (a) Displacement in the $y$ direction, (b) snapshot at $\bar{t}=424$ and $\bar{z}=190-210$, and (c) snapshot at $\bar{t}=1058$ and $\bar{z}=490-510$.

Fig. 12. Waveforms at six positions and displacement distributions for input loading of $\bar{f}_{c}=1$. (a) Displacement in the $y$ direction, and (b) snapshot at $\bar{t}=179$ and $\bar{z}=190-210$. 


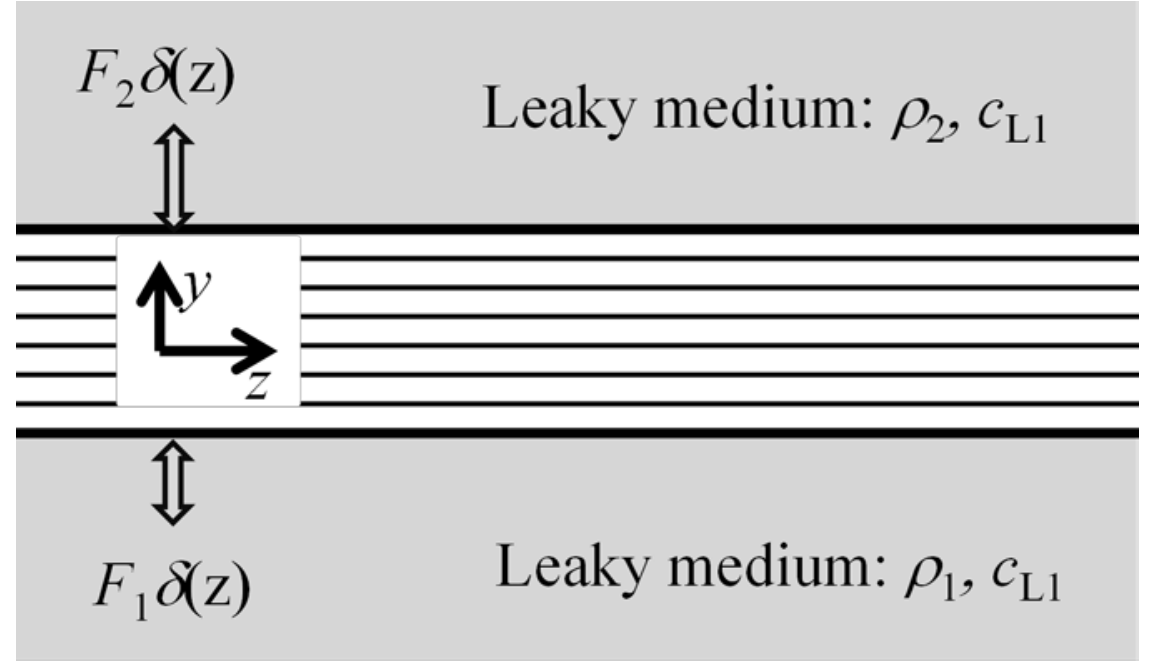

Fig.1. 
Eq. (7)

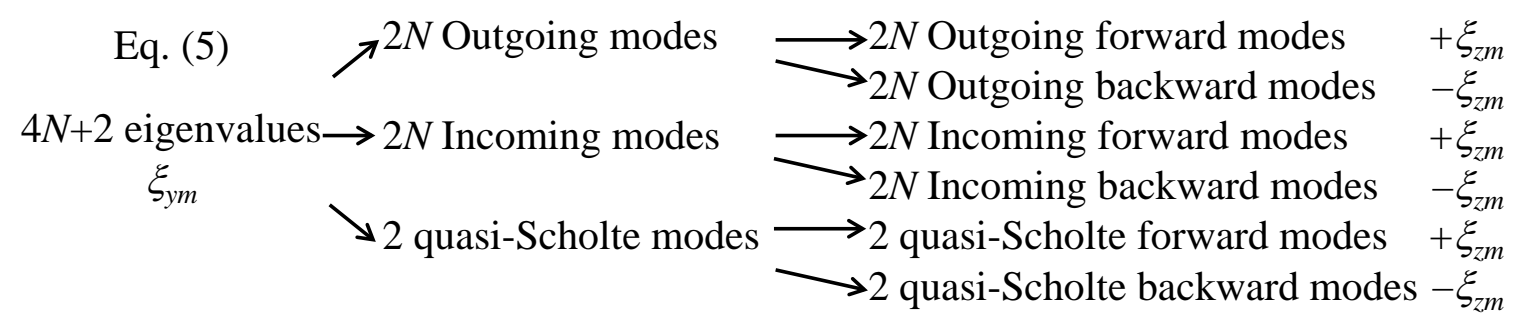

Fig. 2. 

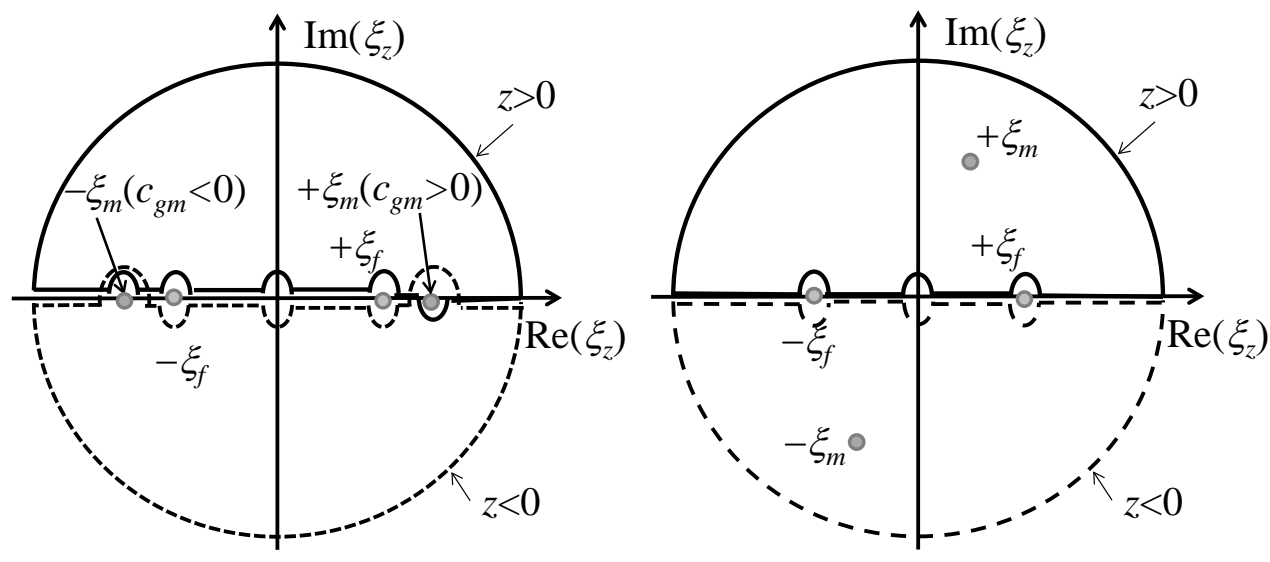

(a)

(b)

Fig. 3. 


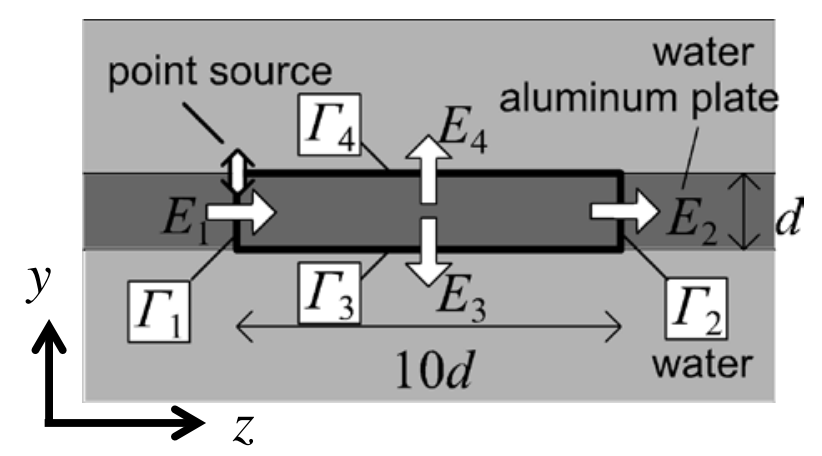

(a)

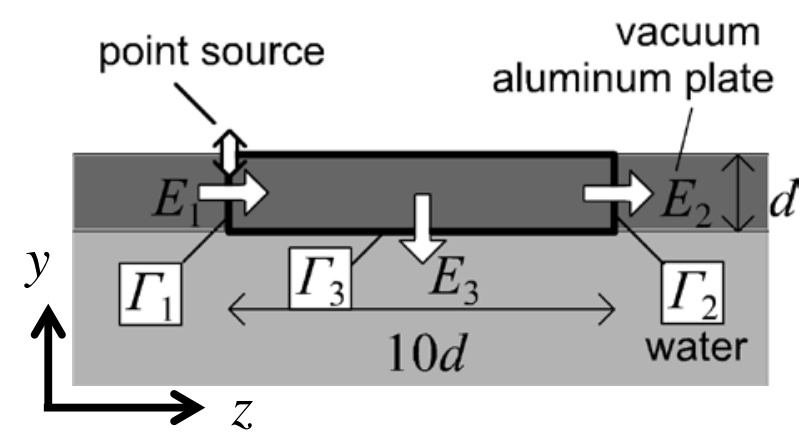

(b)

Fig. 4. 


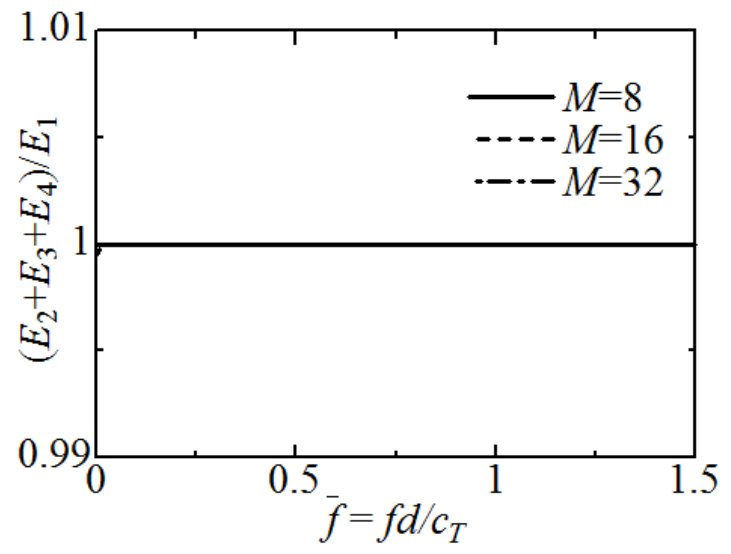

(a)

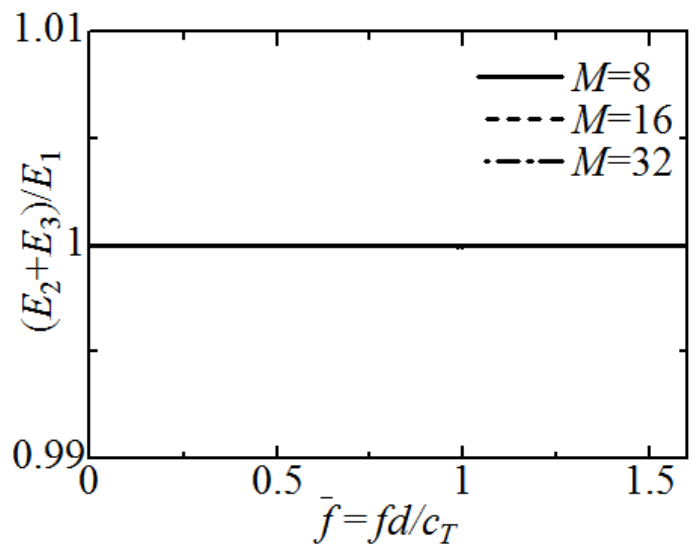

(b)

Fig. 5. 


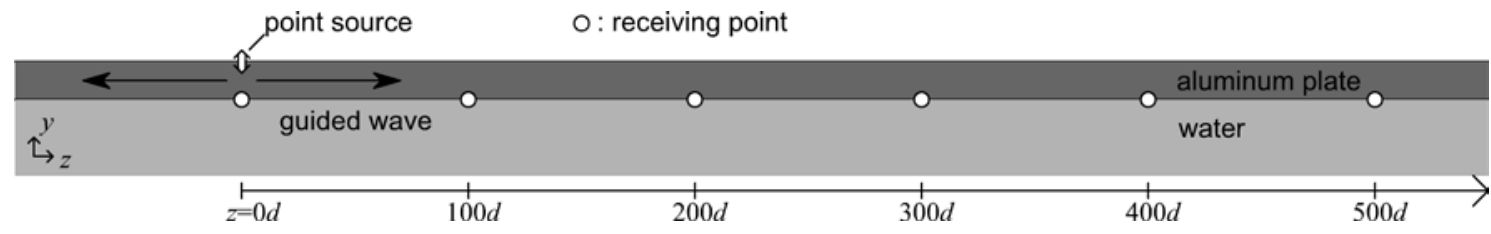

Fig. 6. 


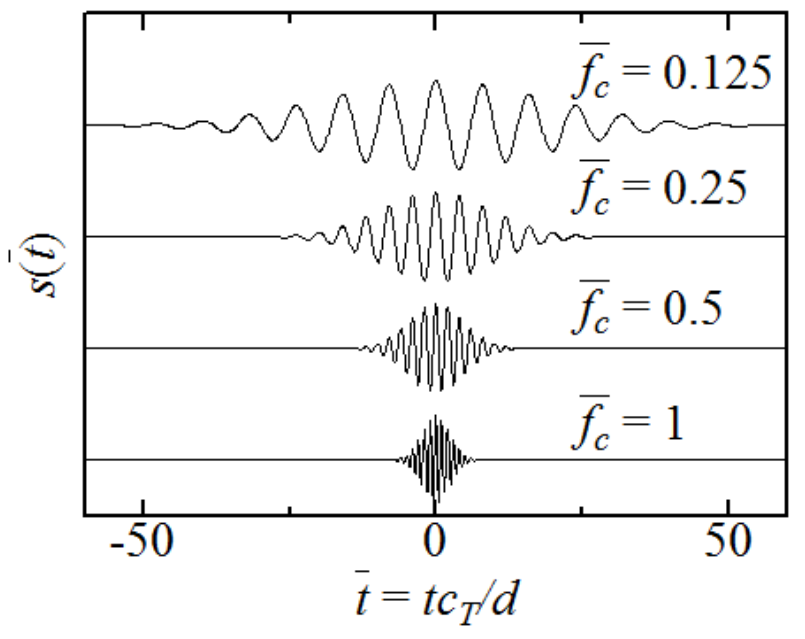

Fig. 7. 


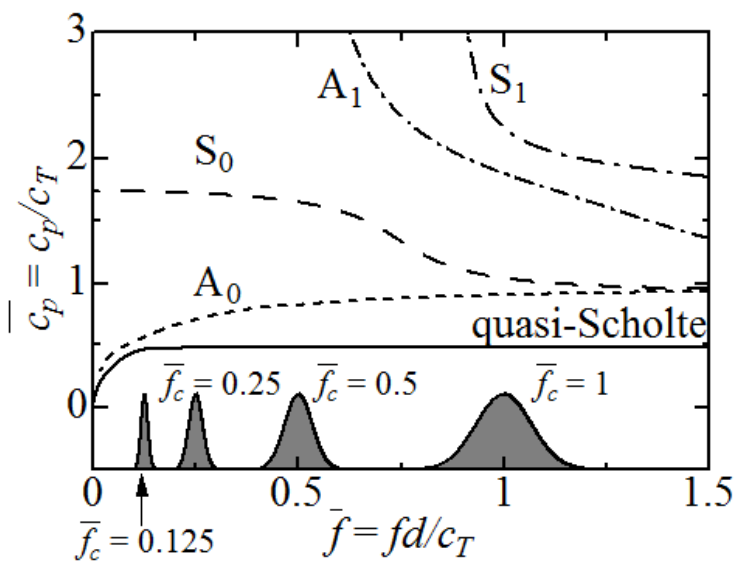

(a)

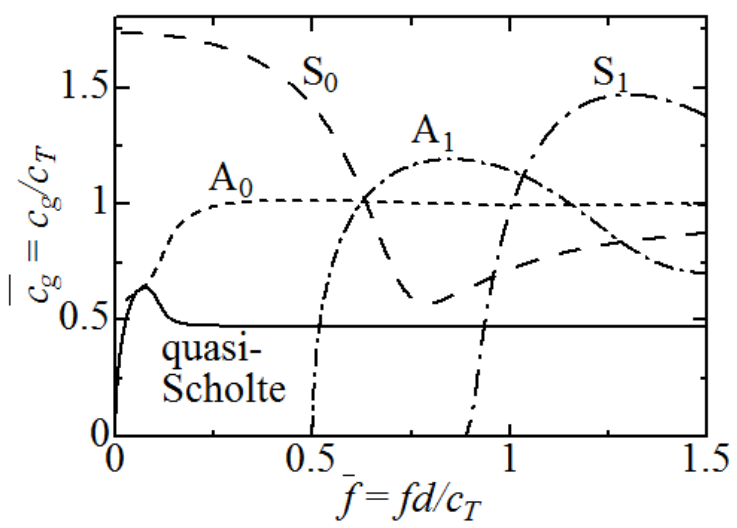

(b)

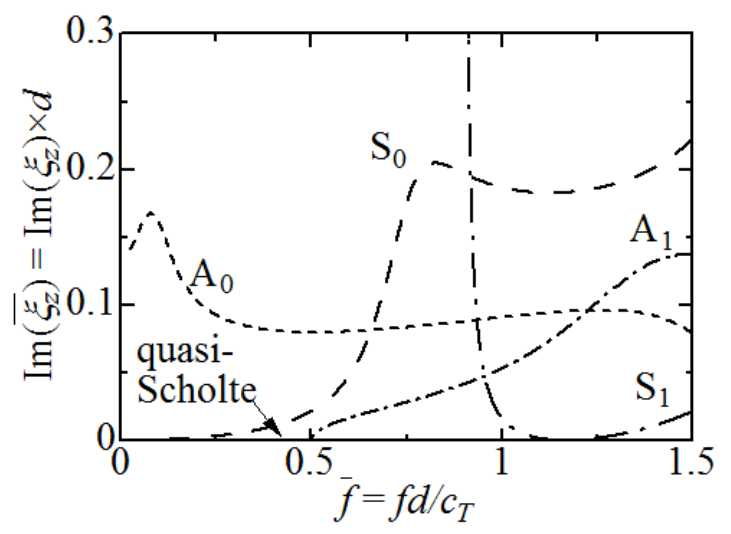

(c)

Fig. 8. 


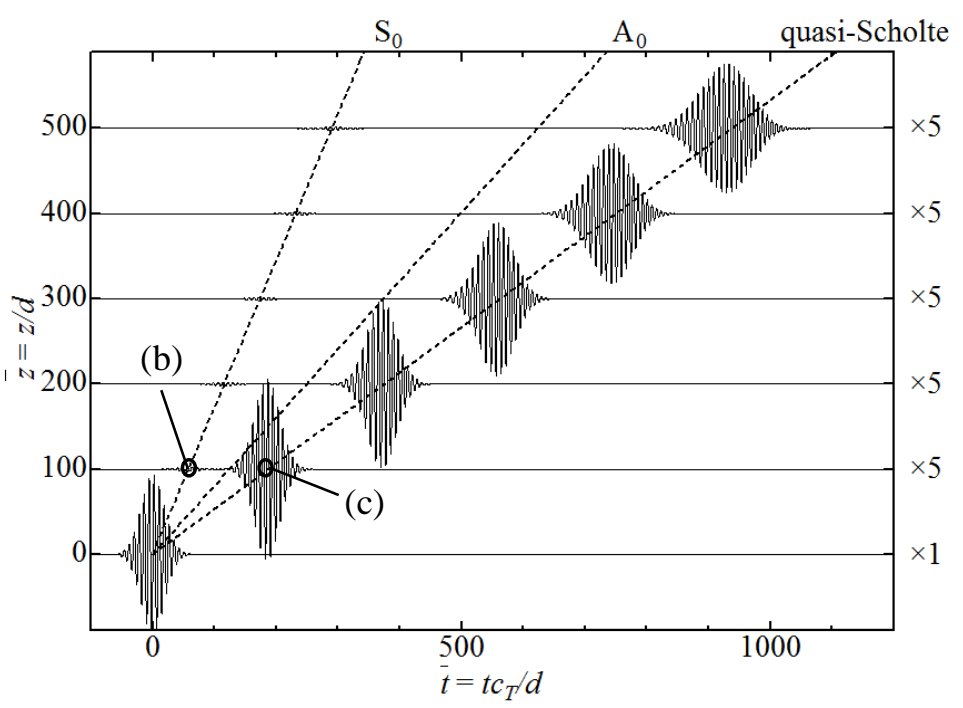

(a)

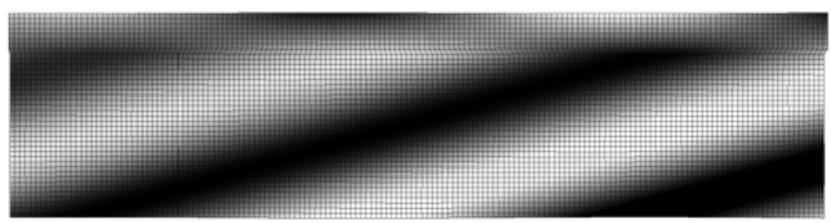

(b)

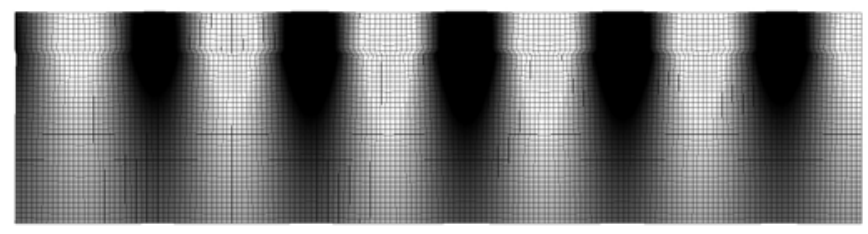

(c)

Fig. 9. 


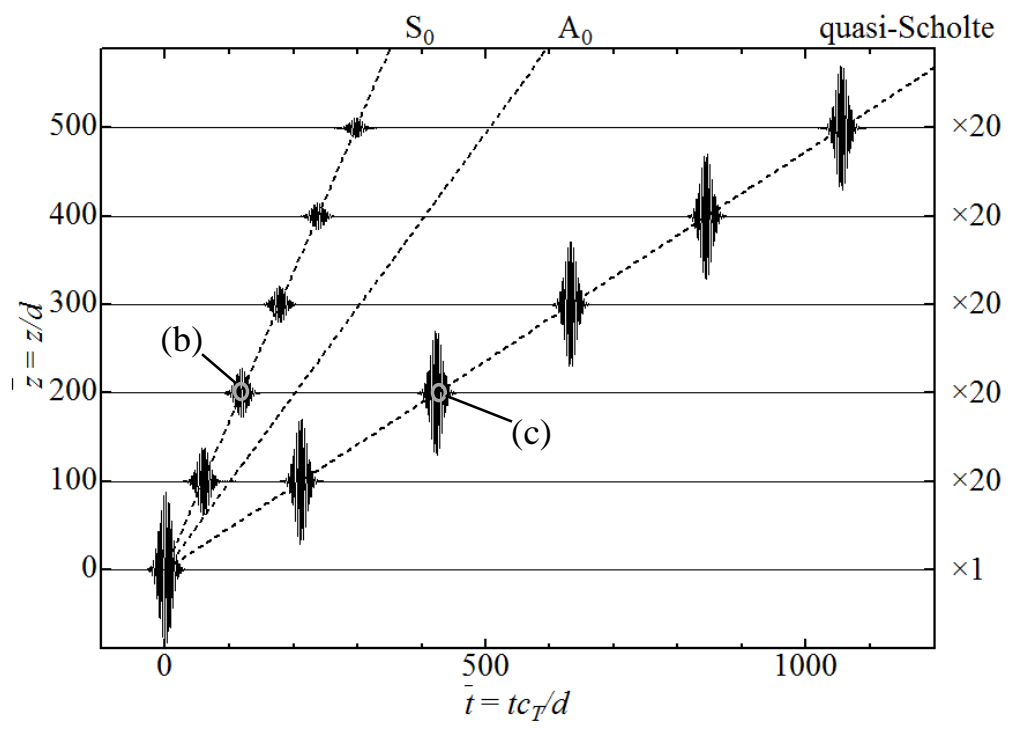

(a)

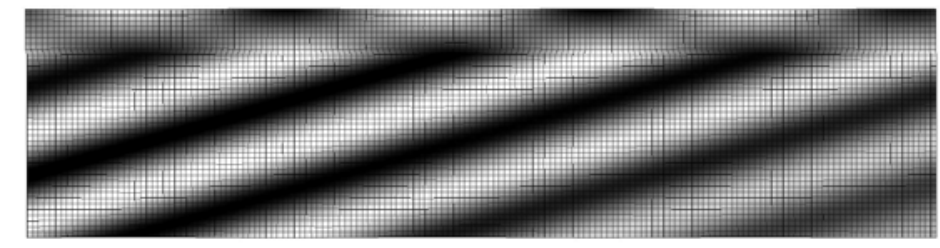

(b)

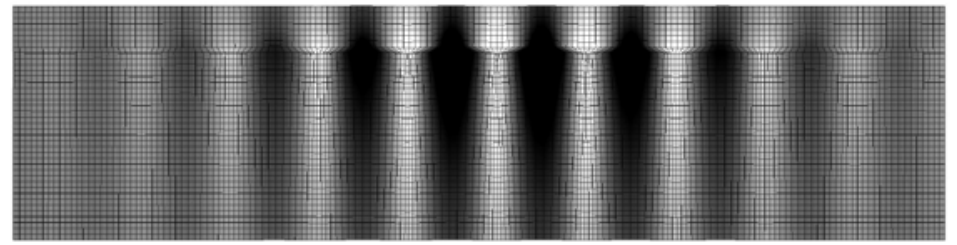

(c)

Fig. 10. 


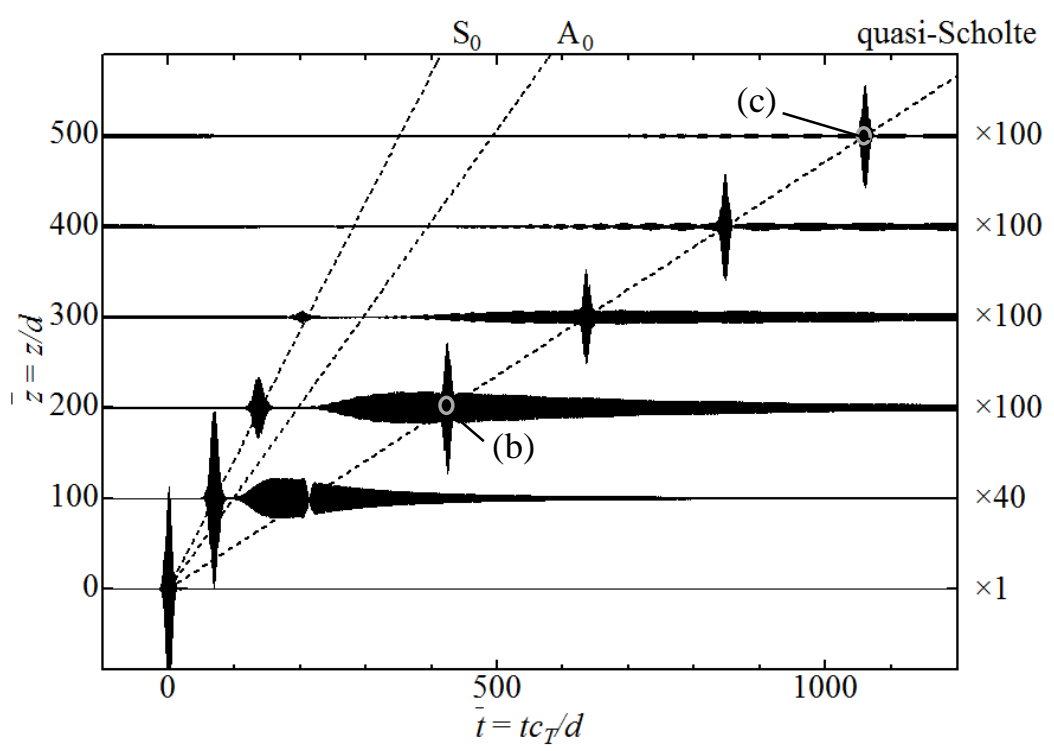

(a)

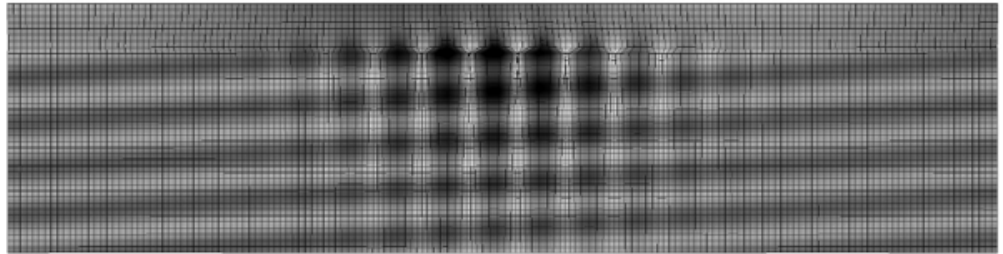

(b)

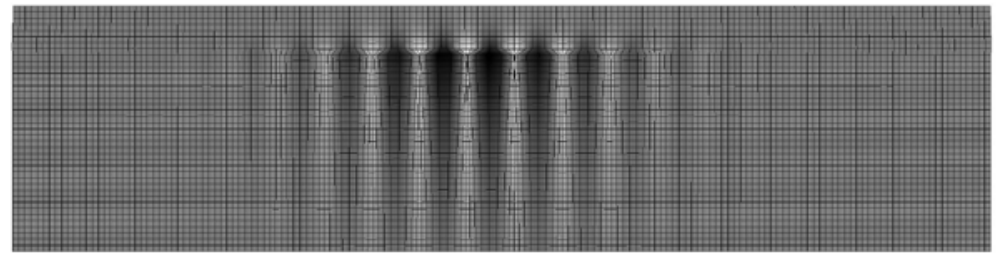

(c)

Fig. 11. 


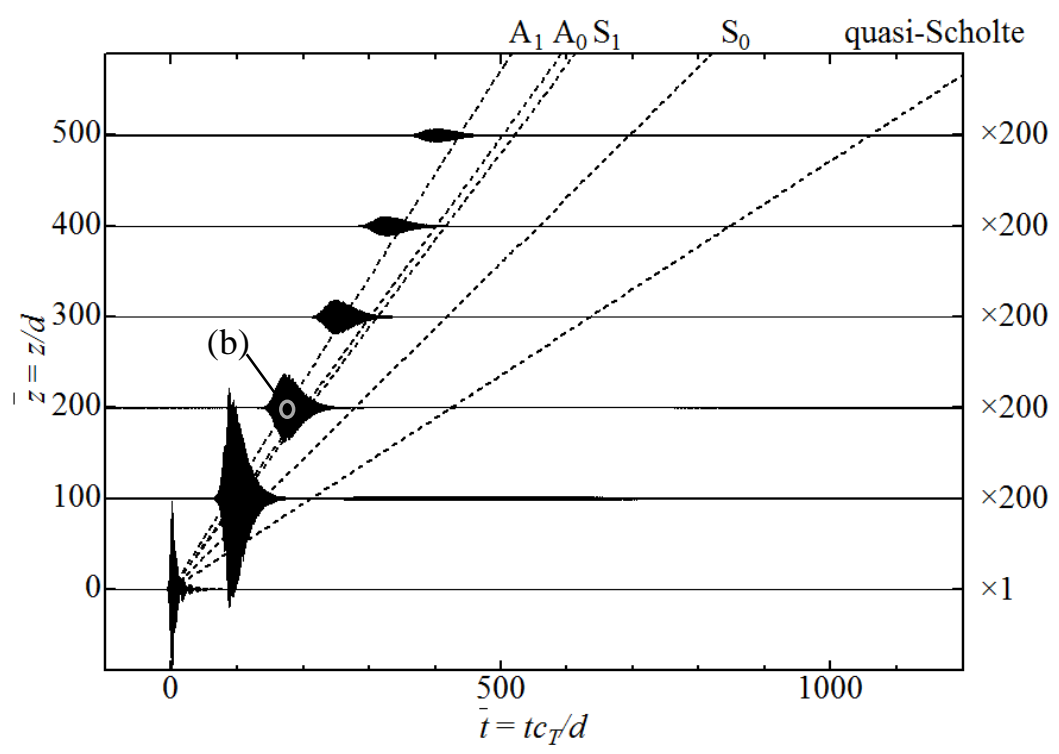

(a)

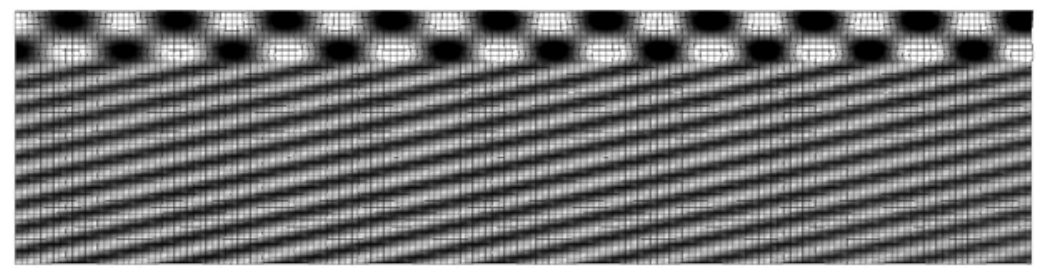

(b)

Fig. 12. 